\title{
Generalized TMDs in the exclusive double Drell-Yan process
}

\section{Shohini Bhattacharya*}

Department of Physics, SERC, Temple University, Philadelphia, PA 19122, USA

E-mail: tug23108@temple.edu

\section{Andreas Metz}

Department of Physics, SERC, Temple University, Philadelphia, PA 19122, USA

E-mail: metza@temple.edu

\section{Jian Zhou}

School of Physics and Key Laboratory of Particle Physics and Particle Irradiation (MOE), Shandong University, Jinan, Shandong 250100, China

E-mail: zhoujiansdu@mail.sdu.edu.cn

Generalized TMDs (GTMDs) of hadrons are the most general two-parton correlation functions. Upon certain projections, several GTMDs reduce to generalized parton distributions (GPDs) and transverse momentum dependent parton distributions (TMDs), respectively. Therefore, GTMDs can be considered as partonic "mother functions". Moreover, two of the GTMDs play an important role in the nucleon spin structure. We show that, by means of the exclusive double Drell-Yan process, GTMDs for quarks can in principle be measured. This is the first known process which is sensitive to these objects. We argue that specific GTMDs can be addressed via suitable polarization observables. We also identify other processes that are directly sensitive to GTMDs.

XXV International Workshop on Deep-Inelastic Scattering and Related Subjects

3-7 April 2017

University of Birmingham, $U K$

\footnotetext{
*Speaker.
} 


\section{Introduction}

Multi-dimensional imaging of strongly interacting systems is currently a very active research area. The key quantities of this field are GPDs and TMDs - generalizations of the one-dimensional parton distributions (PDFs), which provide multi-dimensional images of hadrons in position space and momentum space, respectively. In this context, also GTMDs [1, 2, 3] have recently attracted a lot of interest. Since several GTMDs reduce to GPDs and TMDs in certain kinematical limits, they are often denoted as "mother distributions." Moreover, two of the GTMDs $-F_{1,4}$ and $G_{1,1}$ in the notation of [2] - play a crucial role for the nucleon spin structure. Both functions describe the strength of spin-orbit interactions that are similar to those in a hydrogen atom $[4,5]$. There is also a direct relation between $F_{1,4}$ and the orbital angular momentum (OAM) of partons inside a longitudinally polarized nucleon $[4,6,7]$. It is remarkable that the same relation between $F_{1,4}$ and the quark OAM holds for both commonly used OAM definitions - the (canonical) one by Jaffe and Manohar $\left(L_{\mathrm{JM}}\right)$ [8], and the one by $\mathrm{Ji}\left(L_{\mathrm{Ji}}\right)$ [9]. Moreover, it gives access to the so far elusive $L_{\mathrm{JM}}$ in quantum chromodynamics (QCD) on the lattice $[6,10]$.

While a number of model calculations of GTMDs is available by now, for many years it was not known how GTMDs can be measured. Only recently it was shown that gluon GTMDs can be accessed via diffractive di-jet production in deep-inelastic lepton-nucleon/nucleus scattering [11, $12,13]$, and virtual photon-nucleus quasi-elastic scattering [14]. It was also pointed out that gluon GTMDs can be studied in proton-nucleus collisions [15]. With the exception of [13], the papers on observables for GTMDs deal with the small- $x$ region of parton saturation.

We identify, for the first time, a physical process which gives access to quark GTMDs [16]. Specifically, we show how they enter the exclusive pion-nucleon double Drell-Yan process, $\pi N \rightarrow$ $\left(\ell_{1}^{-} \ell_{1}^{+}\right)\left(\ell_{2}^{-} \ell_{2}^{+}\right) N^{\prime}$, where in the final state one has two di-lepton pairs plus a nucleon. We perform a leading-order (LO) analysis in perturbative QCD. The GTMDs $F_{1,4}$ and $G_{1,1}$ can be measured through suitable polarization observables, and other quark GTMDs could be systematically studied in the same process.

\section{Generalized TMDs}

Let us first recall the definition of quark GTMDs for a nucleon [1, 2]. They parameterize the off-forward transverse momentum dependent quark-quark correlator

$$
W_{\lambda, \lambda^{\prime}}^{q[\Gamma]}\left(P, \Delta, x, \vec{k}_{\perp}\right)=\left.\int \frac{d z^{-} d^{2} \vec{z}_{\perp}}{2(2 \pi)^{3}} e^{i k \cdot z}\left\langle p^{\prime}, \lambda^{\prime}\left|\bar{q}\left(-\frac{z}{2}\right) \Gamma \mathscr{W}\left(-\frac{z}{2}, \frac{z}{2}\right) q\left(\frac{z}{2}\right)\right| p, \lambda\right\rangle\right|_{z^{+}=0},
$$

where $q$ indicates the quark flavor and $\Gamma$ a generic gamma matrix. The 4-momenta and the helicities of the incoming (outgoing) nucleon are denoted by $p\left(p^{\prime}\right)$ and $\lambda\left(\lambda^{\prime}\right)$, respectively. We also use the definitions $P=\left(p+p^{\prime}\right) / 2$ and $\Delta=p^{\prime}-p$. The two quark fields of the operator in (2.1) are separated along the light-cone minus direction $z^{-}$and the transverse direction $\vec{z}_{\perp}$. (We define the light-cone components of a generic 4-vector $a=\left(a^{0}, a^{1}, a^{2}, a^{3}\right)$ through $a^{ \pm}=\left(a^{0} \pm a^{3}\right) / \sqrt{2}$ and $\vec{a}_{\perp}=\left(a^{1}, a^{2}\right)$.) The Wilson line $\mathscr{W}$ makes the bi-local operator color gauge invariant. The average longitudinal and transverse quark momenta are given by $x$ and $\vec{k}_{\perp}$, respectively. 
Here we need the parametrization of (2.1) in terms of GTMDs for $\Gamma=\gamma^{+}, \gamma^{+} \gamma_{5}$. In the case of $\gamma^{+}$one has [2]

$$
\begin{array}{r}
W_{\lambda, \lambda^{\prime}}^{q\left[\gamma^{+}\right]}=\frac{1}{2 M} \bar{u}\left(p^{\prime}, \lambda^{\prime}\right)\left[F_{1,1}^{q}+\frac{i \sigma^{i+} k_{\perp}^{i}}{P^{+}} F_{1,2}^{q}+\frac{i \sigma^{i+} \Delta_{\perp}^{i}}{P^{+}} F_{1,3}^{q}+\frac{i \sigma^{i j} k_{\perp}^{i} \Delta_{\perp}^{j}}{M^{2}} F_{1,4}^{q}\right] u(p, \lambda) \\
=\frac{1}{M \sqrt{1-\xi^{2}}}\left\{\left[M \delta_{\lambda, \lambda^{\prime}}-\frac{1}{2}\left(\lambda \Delta_{\perp}^{1}+i \Delta_{\perp}^{2}\right) \delta_{\lambda,-\lambda^{\prime}}\right] F_{1,1}^{q}+\left(1-\xi^{2}\right)\left(\lambda k_{\perp}^{1}+i k_{\perp}^{2}\right) \delta_{\lambda,-\lambda^{\prime}} F_{1,2}^{q}\right. \\
\left.\left(1-\xi^{2}\right)\left(\lambda \Delta_{\perp}^{1}+i \Delta_{\perp}^{2}\right) \delta_{\lambda,-\lambda^{\prime}} F_{1,3}^{q}+\frac{i \varepsilon_{\perp}^{i j} k_{\perp}^{i} \Delta_{\perp}^{j}}{M^{2}}\left[\lambda M \delta_{\lambda, \lambda^{\prime}}-\frac{\xi}{2}\left(\Delta_{\perp}^{1}+i \lambda \Delta_{\perp}^{2}\right) \delta_{\lambda,-\lambda^{\prime}}\right] F_{1,4}^{q}\right\} .
\end{array}
$$

To evaluate the first line of Eq. (2.2) we considered $u(p, \lambda)$ and $u\left(p^{\prime}, \lambda^{\prime}\right)$ as light-cone helicity spinors (see, e.g., Ref. [17]). Also, $M$ is the nucleon mass, $\xi=\left(p^{+}-p^{\prime+}\right) /\left(p^{+}+p^{\prime+}\right)=$ $-\Delta^{+} /\left(2 P^{+}\right)$the longitudinal momentum transfer to the nucleon, $\sigma^{\mu v}=i\left[\gamma^{\mu}, \gamma^{v}\right] / 2$, and $\varepsilon_{\perp}^{i j}=\varepsilon^{-+i j}$ with $\varepsilon^{0123}=1$. For a generic GTMD one has $X=X\left(x, \xi, \vec{k}_{\perp}, \vec{\Delta}_{\perp}\right)$, where the dependence on $\vec{k}_{\perp}$ and $\vec{\Delta}_{\perp}$ is through the allowed scalar products of these vectors. Note that GTMDs are complex-valued functions [1,2]. Analogous to (2.2), the correlator $W_{\lambda, \lambda^{\prime}}^{q\left[\gamma^{+} \gamma_{5}\right]}$ is also parameterized through four GTMDs, denoted by $G_{1, i}(i=1,2,3,4)$ in Ref. [2]. As pointed out above, our main focus will be on the GTMDs $F_{1,4}$ and $G_{1,1}$. The real part of the GTMDs $F_{1,1}$ and $G_{1,4}$ is related to the distribution of unpolarized quarks in an unpolarized nucleon and the distribution of longitudinally polarized quarks in a longitudinally polarized nucleon, respectively $[2,4]$. Since these distributions are large we will also consider observables which are sensitive to their interference with $F_{1,4}$ and $G_{1,1}$. The double Drell-Yan process implies in Eq. (2.1) a staple-like past-pointing Wilson line [18], identical to the one that appears in TMD factorization of the ordinary Drell-Yan process [19].

\section{Double Drell-Yan process and polarization observables}

To calculate observables we consider the production of two virtual photons rather than two di-lepton pairs. Specifically, we study the process

$$
\pi\left(p_{b}\right)+N\left(p_{a}, \lambda_{a}\right) \rightarrow \gamma_{1}^{*}\left(q_{1}, \lambda_{1}\right)+\gamma_{2}^{*}\left(q_{2}, \lambda_{2}\right)+N^{\prime}\left(p_{a}^{\prime}, \lambda_{a}^{\prime}\right)
$$

From here on the variables of the incoming and outgoing nucleon carry an index $a$. We concentrate on large $s=\left(p_{a}+p_{b}\right)^{2} \approx 2 p_{a}^{+} p_{b}^{-}$, large photon virtualities $q_{1}^{2}, q_{2}^{2}$, and small transverse photon momenta, $\left|\vec{q}_{i \perp}^{2}\right| \ll q_{i}^{2}$. In this region one can use TMD factorization. The longitudinal momentum transfer to the nucleon is given by $\xi_{a}=\left(q_{1}^{+}+q_{2}^{+}\right) /\left(2 P_{a}^{+}\right)$. The LO diagrams for this process are shown in Fig.1. The scattering amplitude depends on the helicities of the nucleons and photons,

$$
\mathscr{T}_{\lambda_{a}, \lambda_{a}^{\prime}}^{\lambda_{1}, \lambda_{2}}=\mathscr{T}_{\lambda_{a}, \lambda_{a}^{\mu}}^{\mu v} \varepsilon_{\mu}^{*}\left(\lambda_{1}\right) \varepsilon_{v}^{*}\left(\lambda_{2}\right)
$$

where $\varepsilon^{\mu}\left(\lambda_{1}\right)$ and $\varepsilon^{\mu}\left(\lambda_{2}\right)$ are the photon polarization vectors. One finds [16]

$$
\begin{aligned}
\mathscr{T}_{\lambda_{a}, \lambda_{a}^{\prime}}^{\mu v}= & i \sum_{q, q^{\prime}} e_{q} e_{q}^{\prime} e^{2} \frac{1}{N_{c}} \int d^{2} \vec{k}_{a \perp} \int d^{2} \vec{k}_{b \perp} \delta^{(2)}\left(\frac{\Delta \vec{q}_{\perp}}{2}-\vec{k}_{a \perp}-\vec{k}_{b \perp}\right) \Phi_{\pi}^{q^{\prime} q}\left(x_{b}, \vec{k}_{b \perp}^{2}\right) \\
& {\left[-i \varepsilon_{\perp}^{\mu v}\left(W_{\lambda_{a}, \lambda_{a}^{\prime}}^{q q^{\prime}\left[\gamma^{+}\right]}\left(x_{a}, \vec{k}_{a \perp}\right)-W_{\lambda_{a}, \lambda_{a}^{\prime}}^{q q^{\prime}\left[\gamma^{+}\right]}\left(-x_{a},-\vec{k}_{a \perp}\right)\right)\right.} \\
& \left.-g_{\perp}^{\mu v}\left(W_{\lambda_{a}, \lambda_{a}^{\prime}}^{q q^{\prime}\left[\gamma^{+} \gamma_{5}\right]}\left(x_{a}, \vec{k}_{a \perp}\right)+W_{\lambda_{a}, \lambda_{a}^{\prime}}^{q \gamma^{\prime}\left[\gamma^{+} \gamma_{5}\right]}\left(-x_{a},-\vec{k}_{a \perp}\right)\right)\right],
\end{aligned}
$$




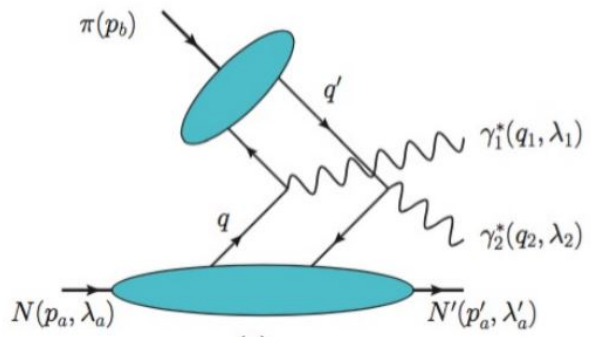

(a)

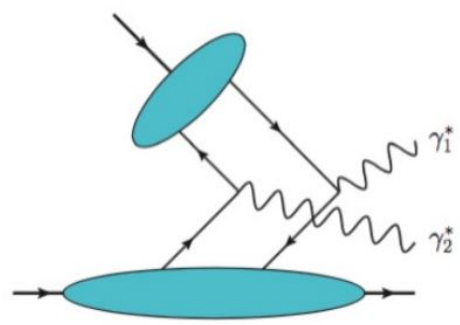

(b)

Figure 1: LO diagrams for the exclusive double Drell-Yan process.

where $e_{q}$ and $e_{q}^{\prime}$ are the quark charges in units of the elementary charge $e$. The expression in (3.3) describes the double Drell-Yan process for all possible pion and nucleon charge states. Note that $\Phi_{\pi}^{q^{\prime} q}$ is the light-cone wave function as defined in [17] for instance, but with the operator $\bar{q}^{\prime} \gamma^{-} \gamma_{5} q$. Likewise, $W^{q q^{\prime}[\Gamma]}$ is given by (2.1) with the operator $\bar{q} \Gamma q^{\prime}$. In Eq. (3.3) we use the vector $\Delta \vec{q}_{\perp}=$ $\vec{q}_{1 \perp}-\vec{q}_{2 \perp}$. The transverse momenta of the photons can be expressed by $\Delta \vec{q}_{\perp}$ and the transverse momentum transfer to the nucleon $\vec{\Delta}_{a \perp}=-\left(\vec{q}_{1 \perp}+\vec{q}_{2 \perp}\right)$. The longitudinal quark momenta are fixed according to $x_{a}=\left(q_{1}^{+}-q_{2}^{+}\right) /\left(2 P_{a}^{+}\right), x_{b}=1-q_{1}^{-} / p_{b}^{-}=q_{2}^{-} / p_{b}^{-}$. The value for $x_{a}$ implies the socalled ERBL region [20, 21], characterized by $-\xi_{a} \leq x_{a} \leq \xi_{a}$. From (3.3) one readily sees that the dominant contribution to the amplitude is for transversely polarized photons.

Below we consider the unpolarized cross section, single-spin asymmetries (SSAs), and doublespin asymmetries (DSAs). It is convenient to introduce

$$
\begin{aligned}
\tau_{U U} & =\frac{1}{2} \sum_{\lambda, \lambda^{\prime}}\left|\mathscr{T}_{\lambda, \lambda^{\prime}}\right|^{2}, \\
\tau_{L U} & =\frac{1}{2} \sum_{\lambda^{\prime}}\left(\left|\mathscr{T}_{+, \lambda^{\prime}}\right|^{2}-\left|\mathscr{T}_{-, \lambda^{\prime}}\right|^{2}\right), \\
\tau_{L L} & =\frac{1}{2}\left(\left(\left|\mathscr{T}_{+,+}\right|^{2}-\left|\mathscr{T}_{+,-}\right|^{2}\right)-\left(\left|\mathscr{T}_{-,+}\right|^{2}-\left|\mathscr{T}_{-,-}\right|^{2}\right)\right),
\end{aligned}
$$

where summation over the photon polarizations is implied. Obviously, $\tau_{L U}$ determines the numerator of the longitudinal target SSA, whereas $\tau_{L L}$ describes the longitudinal DSA with polarization of both the target and the recoil nucleon. Spin asymmetries for transverse polarization in the $x$ direction or $y$-direction are defined accordingly.

In order to get direct access to $F_{1,4}$, that is, without interference with other GTMDs, one has to consider a linear combination of (polarization) observables [16],

$$
\begin{aligned}
\frac{1}{4}\left(\tau_{U U}+\tau_{L L}-\tau_{X X}-\tau_{Y Y}\right)= & \frac{2}{M^{4}}\left(\varepsilon_{\perp}^{i j} \Delta q_{\perp}^{i} \Delta_{a \perp}^{j}\right)^{2} C^{(+)}\left[\vec{\beta}_{\perp} \cdot \vec{k}_{a \perp} F_{1,4} \Phi_{\pi}\right] C^{(+)}\left[\vec{\beta}_{\perp} \cdot \vec{k}_{a \perp} F_{1,4}^{*} \Phi_{\pi}^{*}\right] \\
& +2 C^{(+)}\left[G_{1,4} \Phi_{\pi}\right] C^{(+)}\left[G_{1,4}^{*} \Phi_{\pi}^{*}\right] .
\end{aligned}
$$

In Eq. (3.7) we use the shorthand notation

$$
\begin{gathered}
C^{( \pm)}\left[w\left(\vec{k}_{a \perp}, \vec{k}_{b \perp}\right) X \Phi_{\pi}\right]=\frac{e^{2}}{\sqrt{1-\xi_{a}^{2}} N_{c}} \sum_{q, q^{\prime}} e_{q} e_{q}^{\prime} \int d^{2} \vec{k}_{a \perp} \int d^{2} \vec{k}_{b \perp} \delta^{(2)}\left(\frac{\Delta \vec{q}_{\perp}}{2}-\vec{k}_{a \perp}-\vec{k}_{b \perp}\right) w\left(\vec{k}_{a \perp}, \vec{k}_{b \perp}\right) \\
\times\left[X^{q q^{\prime}}\left(x_{a}, \vec{k}_{a \perp}\right) \pm X^{q q^{\prime}}\left(-x_{a},-\vec{k}_{a \perp}\right)\right] \Phi_{\pi}^{q^{\prime} q}\left(x_{b}, \vec{k}_{b \perp}^{2}\right)
\end{gathered}
$$


with $w\left(\vec{k}_{a \perp}, \vec{k}_{b \perp}\right)$ a generic weight function. The vector $\vec{\beta}_{\perp}$ in (3.7) is given by $\Delta \vec{q}_{\perp}$ and $\vec{\Delta}_{a \perp}$ [16]. We repeat that in order to obtain Eq. (3.7) the photon polarizations have been summed over. In Eq. (3.7), in addition to $F_{1,4}$ the GTMD $G_{1,4}$ appears which is presumably large as already mentioned above. However, one can separate the two contributions in (3.7) by not summing over the photon polarizations but rather considering specific linear polarization states [18]. To address $G_{1,1}$ one can study $\frac{1}{4}\left(\tau_{U U}+\tau_{L L}+\tau_{X X}+\tau_{Y Y}\right)$. The result for this linear combination is identical to (3.7), but with the replacements $F_{1,4} \rightarrow G_{1,1}$ and $G_{1,4} \rightarrow F_{1,1}$.

The observable in (3.7), as well as the corresponding observable for $G_{1,1}$, may have a drawback: In these linear combinations there is cancellations of potentially large terms [18]. It may therefore be beneficial to also explore interference between $F_{1,4}$ (or $G_{1,1}$ ) and other GTMDs. Such an interference shows up in the following linear combination of longitudinal SSAs [16]:

$$
\begin{aligned}
\frac{1}{2}\left(\tau_{L U}+\tau_{U L}\right)= & \frac{1}{2}\left(\left|\mathscr{T}_{+,+}\right|^{2}-\left|\mathscr{T}_{-,-}\right|^{2}\right)=\frac{4}{M^{2}} \varepsilon_{\perp}^{i j} \Delta q_{\perp}^{i} \Delta_{a \perp}^{j} \operatorname{Im}\left\{C^{(-)}\left[F_{1,1} \Phi_{\pi}\right] C^{(+)}\left[\vec{\beta}_{\perp} \cdot \vec{k}_{a \perp} F_{1,4}^{*} \Phi_{\pi}^{*}\right]\right. \\
& \left.-C^{(+)}\left[G_{1,4} \Phi_{\pi}\right] C^{(-)}\left[\vec{\beta}_{\perp} \cdot \vec{k}_{a \perp} G_{1,1}^{*} \Phi_{\pi}^{*}\right]\right\} .
\end{aligned}
$$

Further polarization observables exist which involve interference between $F_{1,4}$ (or $G_{1,1}$ ) and other GTMDs, but the one in (3.9) gives the simplest expression [18]. On the r.h.s. of (3.9) the imaginary part of products of GTMDs appears. According to current knowledge the GTMDs most relevant for the spin structure of the nucleon are $\operatorname{Re} F_{1,4}$ and $\operatorname{Re} G_{1,1}$. Though these functions contribute to (3.9), they interfere with $\operatorname{Im} F_{1,4}$ and $\operatorname{Im} G_{1,1}$, respectively. At present, there exists no information on the latter functions, and they may in fact be small. This issue can be overcome by considering the observable $\frac{1}{2}\left(\tau_{X Y}-\tau_{Y X}\right)$, whose result agrees with the r.h.s. of (3.9) but with $\operatorname{Re}\{\ldots\}$ instead of $\operatorname{Im}\{\ldots\}[16]$.

\section{Summary and Outlook}

We have shown that quark GTMDs can be studied through the exclusive double Drell-Yan process. To leading order in perturbative QCD, this process in sensitive to GTMDs in the ERBL region. The main focus was on the GTMDs $F_{1,4}$ and $G_{1,1}$, which attracted much attention because of their relation to the spin structure of the nucleon. We have proposed several polarization observables that can give access to these GTMDs. Other GTMDs could be explored via suitable polarization observables [18].

An attempt should be made to numerically estimate the unpolarized cross section and the various spin asymmetries for $\pi N \rightarrow\left(\ell_{1}^{-} \ell_{1}^{+}\right)\left(\ell_{2}^{-} \ell_{2}^{+}\right) N^{\prime}$. We also note that one can perform a similar analysis for nucleon-nucleon collisions [18]. Moreover, hadronic final states typically give rise to higher count rates. One such example is the process $p p \rightarrow \eta_{c} \eta_{c} p p$, which can basically be treated along the lines discussed above, but gluon GTMDs enter the analysis [18]. Finally, the type of reactions discussed here could also provide constraints on GPDs in the ERBL region.

\section{Acknowledgments}

This work has been supported by the National Science Foundation under Contract No. PHY1516088 (A.M.), the National Science Foundation of China under Grant No. 11675093, and by the 
Thousand Talents Plan for Young Professionals (J.Z.).

\section{References}

[1] S. Meissner, A. Metz, M. Schlegel and K. Goeke, Generalized parton correlation functions for a spin-O hadron, JHEP 0808, 038 (2008).

[2] S. Meissner, A. Metz and M. Schlegel, Generalized parton correlation functions for a spin-1/2 hadron, JHEP 0908, 056 (2009).

[3] C. Lorcé and B. Pasquini, Structure analysis of the generalized correlator of quark and gluon for a spin-1/2 target, JHEP 1309, 138 (2013).

[4] C. Lorcé and B. Pasquini, Quark Wigner distributions and orbital angular momentum, Phys. Rev. D 84, 014015 (2011).

[5] C. Lorcé, Spin-orbit correlations in the nucleon, Phys. Lett. B 735, 344 (2014).

[6] Y. Hatta, Notes on the orbital angular momentum of quarks in the nucleon, Phys. Lett. B 708, 186 (2012).

[7] X.-D. Ji, X. Xiong and F. Yuan, Proton spin structure from measurable parton distributions, Phys. Rev. Lett. 109, 152005 (2012).

[8] R. L. Jaffe and A. Manohar, The G(1) problem: fact and fantasy on the spin of the proton, Nucl. Phys. B 337, 509 (1990).

[9] X.-D. Ji, Gauge-invariant decomposition of nucleon spin, Phys. Rev. Lett. 78, 610 (1997).

[10] M. Engelhardt, Quark orbital dynamics in the proton from Lattice QCD - from Ji to Jaffe-Manohar orbital angular momentum, Phys. Rev. D 95, 094505 (2017).

[11] Y. Hatta, B. W. Xiao and F. Yuan, Probing the small-x gluon tomography in correlated hard diffractive dijet production in deep-inelastic scattering, Phys. Rev. Lett. 116, 202301 (2016).

[12] Y. Hatta, Y. Nakagawa, B. W. Xiao, F. Yuan and Y. Zhao, Gluon orbital angular momentum at small $x$, arXiv:1612.02445 [hep-ph].

[13] X. Ji, F. Yuan and Y. Zhao, Hunting the gluon orbital angular momentum at the Electron-Ion Collider, Phys. Rev. Lett. 118, 192004 (2017).

[14] J. Zhou, Elliptic gluon generalized transverse-momentum-dependent distribution inside a large nucleus, Phys. Rev. D 94, 114017 (2016).

[15] Y. Hagiwara, Y. Hatta, B. W. Xiao and F. Yuan, Elliptic flow in pA collisions due to elliptic gluon distributions?, arXiv:1701.04254 [hep-ph].

[16] S. Bhattacharya, A. Metz and J. Zhou, Generalized TMDs and the exclusive double Drell-Yan process, arXiv: 1702.04387 [hep-ph].

[17] M. Diehl, Generalized parton distributions, Phys. Rept. 388, 41 (2003).

[18] S. Bhattacharya, A. Metz, J. Zhou, in preparation.

[19] J. C. Collins, Leading-twist single transverse-spin asymmetries: Drell-Yan and deep-inelastic scattering, Phys. Lett. B 536, 43 (2002).

[20] A. V. Efremov and A. V. Radyushkin, Factorization and asymptotical behavior of pion form factor in $Q C D$, Phys. Lett. 94B, 245 (1980).

[21] G. P. Lepage and S. J. Brodsky, Exclusive processes in quantum chromodynamics: evolution equations for hadronic wave functions and the form factors of mesons, Phys. Lett. 87B, 359 (1979). 